\title{
BLUE ELLIPTICALS IN COMPACT GROUPS
}

\author{
Stephen E. Zepf \\ Space Telescope Science Institute and the Johns Hopkins University \\ and \\ Bradley C. Whitmore \\ Space Telescope Science Institute
}

\begin{abstract}
We examine the hypothesis that mergers of spiral galaxies make elliptical galaxies by studying galaxies in compact groups. We combine dynamical models of the merger-rich compact group environment with stellar evolution models and predict that roughly $15 \%$ of compact group ellipticals should be 0.15 mag bluer in $B-R$ color than normal ellipticals. The published colors of these galaxies suggest the existence of this predicted blue population, but a normal distribution with large random errors can not be ruled out based on these data alone. However, we have new $U B V R I$ data which confirm the blue color of the two ellipticals with blue $B-R$ colors for which we have our own colors. This confirmation of a population of blue ellipticals indicates that interactions are occurring in compact groups, but a blue color in one index alone does not require that these ellipticals are recent products of the merger of two spirals. We demonstrate how optical spectroscopy in the blue may distinguish between a true spiral + spiral merger and the swallowing of a gas-rich system by an already formed elliptical. We also show that the sum of the luminosity of the galaxies in each group is consistent with the hypothesis that the final stage in the evolution of a compact group is an elliptical galaxy.
\end{abstract}

\section{Introduction}

Toomre and Toomre's (1972) hypothesis that mergers play a fundamental role in determining the morphology of a galaxy has continued to gain support both from observations of nearby merging systems and from $\mathrm{N}$-body simulations. However, as first noted by Ivan King (1977), the evolutionary sequence from ongoing merger to normal elliptical suffers from a lack of transitional cases. Most studies addressing the "merger hypothesis" have concentrated on systems in which tails and other morphological features indicative of a spiral + spiral merger are still present, but unfortunately the final state of these systems is not conclusively an elliptical galaxy.

We are testing the "merger hypothesis" in a direct way by studying elliptical galaxies in the Hickson compact groups (Hickson 1982). These groups are regions of high spatial density and low relative velocity dispersion where recent interactions and mergers should be most common. The rapid merging of galaxies in such environments is graphically demonstrated by the $\mathrm{N}$ body simulations of compact groups of galaxies by Barnes (1989). These simulations indicate that the time scale for two spirals to collapse into an elliptical looking system is shorter than the time scale for the stellar population of two spirals to evolve to the red color of elliptical galaxies. Therefore, the merger hypothesis predicts that recently merged spirals should have the morphology of an elliptical, but with significantly bluer color than normal ellipticals, both because of the younger population of the merged spirals and because of the burst of star formation associated with the merger. 


\section{Models}

In order to make more detailed predictions about the fraction of compact group ellipticals expected to be blue, we have combined dynamical models of compact group evolution (Barnes 1989) with models of the optical colors of stellar populations (Larson and Tinsley 1978), and . the simple assumption that mergers of spiral form ellipticals. Based on these models, we predict that about $15 \%$ of the ellipticals should be bluer than $(B-V)_{T}^{0}<0.80$, compared to $(B-V)_{T}^{0}=0.90$ typical of normal ellipticals (details of the models are discussed in Zepf and Whitmore 1990).

\section{Optical Colors}

Our first step in this project was to test the prediction of the existence of a population of unusually blue ellipticals in compact groups by comparing the distribution of $B-R$ colors (Hickson et al. 1989) to those in a general sample of early-type galaxies (Sandage and Visvanathan 1978). We find (Zepf and Whitmore 1989, and Figure 1) that a substantial population of blue ellipticals exists in the compact groups, but several suspicious effects present in these $B-R$ colors suggest caution in interpreting these data. For example, the stars in Figure $1 \mathrm{~b}$ represent ellipticals in Hickson groups in which all of the early-type galaxies in that group are abnormally blue, suggesting the possibility of problems with the calibration of the frames of these groups. Even after removing these cases, seven ellipticals are abnormally blue and reside in groups in which at least one galaxy has a color normal for its type (these seven are encircled in Figure $1 \mathbf{b})$.
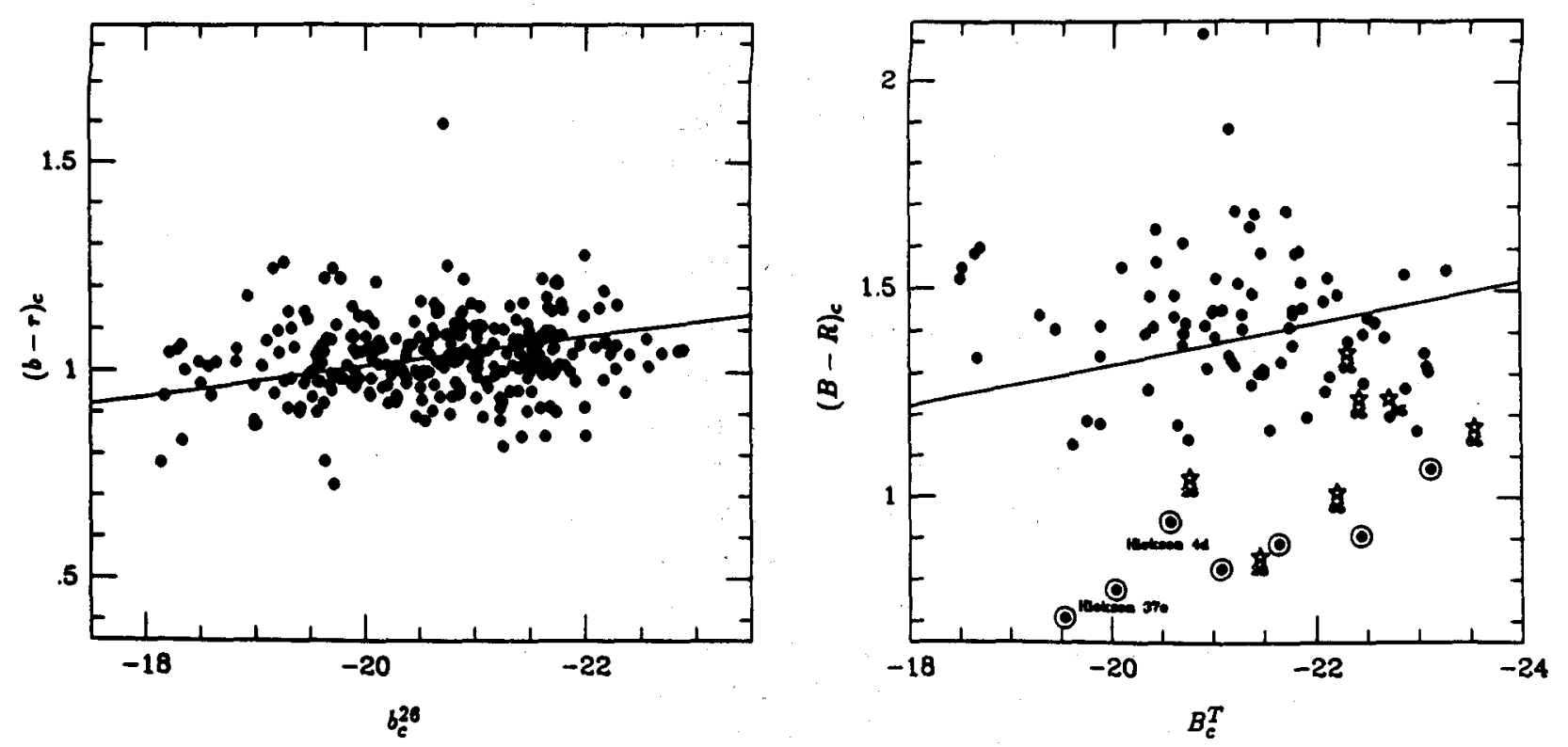

Figure 1. (a) The color-magnitude plot for all of the early-type galaxies in Sandage and Visvanathan (1978). (b) The color-magnitude plot for all of the elliptical galaxies in the Hickson compact groups (Hickson et al. 1989). The seven candidate blue ellipticals are encircled.

Although the $B-R$ colors are suggestive of a population of blue ellipticals in compact groups, a normal distribution of colors with large random errors can not be ruled out based on these date alone. In order to test the reality of the suspected population of blue ellipticals, we undertook an extensive observational program to obtain CCD surface photometry in good 
photometric conditions for a large sample of early-type galaxies in compact groups. We now have obtained $U B V R I$ images for approximately 60 ellipticals in compact groups, completing this aspect of the project. The data reduction is still in progress, but a preliminary result is that for the two cases in which we have reduced our own colors of an elliptical with blue colors in the Hickson et al. data, we confirm their blue color (Hickson 37e $(B-V)_{T}^{0}=0.75$ and Hickson $\left.4 \mathrm{~d}(B-V)_{T}^{0}=0.52\right)$. We also find that the 15 other ellipticals in compact groups which we have reduced have normal colors $\left((B-V)_{T}^{0}=0.91 \pm 0.02\right)$. The discovery that about $15 \%$ of the ellipticals in compact groups are unusually blue, in general agreement with the predictions of our models, represents the major result of this paper.

\section{Spectroscopy}

Even with multicolor data, several different origins are possible for any given blue elliptical. With broad-band colors alone it is difficult to distinguish between an already formed elliptical swallowing a gas rich galaxy and the merger of two spirals creating a new elliptical. As the second step in this project we are undertaking a spectroscopic study of the stellar absorption lines. This spectroscopy directly probes the stellar populations and offers a way to determine the progenitors of the blue ellipticals.

Our spectroscopy centers on the Balmer lines $H \gamma, H \delta$, and $H \epsilon$, and the corresponding nearby metal lines. The young stellar populations of a recent merger of two spiral galaxies will be evident in strong Balmer lines in absorption. Ongoing mergers such as NGC 7252 and NGC 3921 invariably show this signature, which is also observed in about $20 \%$ of the shell galaxies observed by Carter et al. (1989). However, only when one of the progenitors was an already formed elliptical will the blue elliptical have strong metal lines in addition to the strong Balmer lines. Strong Balmer lines and weak metal lines are clearly evident in the spectrum of NGC 7252, a prototypical spiral + spiral merger. Much of our future effort will be devoted to developing more detailed and quantitative tools with which to analyze the spectroscopy (Zepf and Whitmore 1990).

\section{Luminosities of Final Products of Compact Groups}

All of the available data are consistent with the hypothesis that galaxies are merging and forming ellipticals in compact groups. Within the context of the merger hypothesis, each compact group we observe today is destined to become a single elliptical galaxy. Therefore, the sum of the luminosities of the galaxies in the group should be consistent with luminosities of normal ellipticals in equivalently selected samples. Because of the complex selection criteria applied by Hickson to define his compact group sample (Hickson 1982, and this volume), it is difficult to define an exact comparison sample. However, to first-order the compact group sample is equivalent to a magnitude or diameter limited catalog. For the sample of Sadler and Gerhard (1985), which is both magnitude and diameter limited, we find that the mean $M_{B_{0}^{T}}=-21.2$ and the median $M_{B_{0}^{T}}=-21.7$, and the magnitude limited RSA (Sandage and Tamman 1987) sample gives approximately the same value (mean $M_{B_{0}^{T}}=-21.3$ ).

The simplest way to calculate the expected luminosity of the product of the merging of compact group galaxies into a single elliptical is to sum the luminosities of all of the galaxies in each of the groups. This summation gives a mean $M_{B_{0}^{T}}=-21.9$ and a median of $M_{B_{0}^{T}}=-22.6$. A more realistic approach is to model the aging of the stellar populations after the galaxies have merged. Presumably, once the group has coalesced into a single system, star formation will cease, and the resulting galaxy will grow redder in time. We model this evolution by first 
assuming that each galaxy will redden to the color of a elliptical and then use the models of Larson, Tinsley, and Caldwell (1980) to find the resulting fading in $B$. Summing these total, corrected blue luminosities as in the no-evolution case, we find that the mean $M_{B_{0}^{T}}=-21.4$, and the median $M_{B_{0}^{T}}=-21.6$. The similarity of the distribution of the absolute magnitudes in this case with the observed distribution of ellipticals in similarly selected samples is further evidence that the properties of compact groups are consistent with our current understanding of merging and evolution in compact groups.

VI. Conclusions

1. A population of blue ellipticals exists in compact groups.

2. This population of blue ellipticals as well as the total magnitudes of compact group galaxies are consistent with the hypothesis that galaxies in compact groups are merging and forming new ellipticals.

3. Optical spectroscopy may.be able to distinguish a blue elliptical which is the result of the merger of two spirals from an already formed elliptical swallowing a gas rich system.

\section{References}

Barnes, J. 1989, Nature, 338, 123.

Carter, D., Prieur, J.-L., Wilkinson, A., Sparks, W.B., and Malin, D.F. 1988, M. N. R. A. S., 235, 813.

Hickson, P. 1982, Ap. J., 255, 382.

Hickson, P., Kindl, E., and Aumann, J. 1989, Ap. J. Suppl., 70, 687.

King, I.R. 1977, in The Evolution of Galaxies and Stellar Populations, ed. R.B. Larson and B.M. Tinsley (New Haven: Yale University Observatory), p. 418.

Larson, R.B., and Tinsley, B.M. 1978, Ap. J., $219,46$.

Larson, R.B., Tinsley, B.M., and Caldwell, C.N. 1980, Ap. J., 237, 692.

Sadler, E.M., and Gerhard, O.E. 1985, M. N. R. A. S., 214, 177.

Sandage, A., and Visvanathan, V. 1978, Ap. J., $225,742$.

Toomre, A., and Toomre, J. 1972, Ap. J., 178, 623.

Zepf, S.E., and Whitmore, B.C. 1989, in The Interactions and Dynamics of Galaxies, ed. Roland Wielen, in press.

Zepf, S.E., and Whitmore, B.C. 1990, Ap. J., in preparation. 


\section{DISCUSSION}

Struck-Marce11: Could you tell me what burst strengths you used in your models, and whether you think that a SF burst using gas dumped onto a pre-existing elliptical could account for the blue colors?

zepf: A blue color in a single color index (e.g., B-V) can be explained either by a pre-existing elliptical swallowing a gasrich, star-bursting system or by two spirals merging to form a new elliptical. This degeneracy when using a single color index is precisely the reason we use UBVRI colors and optical

spectroscopy.

Hutchings: Do you have a comment on the old E galaxies in the Hickson sample? Could it be dust?

Zepf: The red elliptical galaxies might be explained by dust (as an example, NGC 5128 would appear at the top of this plot). However, the elliptical with the reddest colors in the Hickson et al. data appears to actually have normal colors, with the very red color being due to photometric error. I think we will be able to answer this question better when our photometric survey is complete.

Mamon: The blue ellipticals in your luminosity-color plot of Hickson compact groups tend to be faint, and are often, from the examples you have shown, among the fainter members of their groups. Comment: The statistics on the bright end of the luminosity functions of Hickson's compact groups (based on a test devised by Tremaine and Richstone 1977) show no signs of mergers in these groups. Perhaps the bright-end luminosities are not the ones to study when searching for mergers?!

Zepf: It is certainly true observationally that the blue ellipticals do not seem to be biased to the higher luminosities. 